\section{Padé approximation and linear prediction methods}

SIR-Yeramian and Claverie' ${ }^{1}$ use a method combining the Padé rational approximation and the Laplace transform to extract the coefficients and exponents in multiexponential data. They emphasized three important features: (1) The assumption of the number of decaying exponential components involved in the total signal is not necessary; (2) increased numerical accuracy results if the Taylor series of the Laplace integral is made in the neighbourhood of the actual decay rates; and (3) their method can solve the Lanczos multiple exponential problem. We wish to point out that the above features were already known and could be implemented in the framework of Prony's linear prediction' $^{2}$ (LP) and $z$-transform ${ }^{3}$ theory. More importantly, the LP methods using the Householder decomposition ${ }^{4}$ (LPQRD) or the singular value decomposition (LPSVD) are more stable and accurate than the Longman method ${ }^{6}$ used by them. Both LPQRD and LPSVD methods have been used for some time to extrapolate truncated signals and analyse signals of exponentially damped sinusoid without $a$ priori assumption of the total number of spectral components. The functional form of the Padé approximation is very similar to the linear prediction $z$-transform (LPZ) spectral formula ${ }^{3}$ that we have derived assuming the signal is composed from a finite number of damped sinusoids.

Unlike QRD or SVD, their Longman algorithm ${ }^{\circ}$ may have two limitations: (1) In the Longman approach, the LP coefficients in the Padé table are completely determined from the first $2 M$ data points, where $M$ corresponds to the LP filter length; and (2) The Longman algorithm can be unstable, especially when the first few data points are close to zero. On the other hand, QRD or SVD is favoured because of four points: (1) LPQRD or LPSVD is not subject to signal truncation problems. In contrast, the Laplace integral transform used in ref. 1 can be inaccurate if the signal is severely truncated because the Laplace integral should be evaluated in time from zero to infinity. (2) The decomposition methods work well with singular matrices and have better stability. (3) All data can be used to calculate the LP coefficients, yielding better accuracy than the Longman method which uses fewer data. And (4) QRD or SVD provides eigenvalues and matrix rank determination that can be used to reject noise components effectively. Once the LP coefficients are determined from any of the above methods, the frequencies and the decay rates can be evaluated by solving the roots of the polynomial formed by the LP co-efficients. Finally, the corresponding amplitude and phase for each spectral component can be determined from a linear least-squares fit.

To illustrate the advantages of the
LPQRD method over the Longman method, we present a comparison using a simulated signal of two close components (see table). Fifty data points are generated with a step of $0.1 \mathrm{~s}$, where data values are rounded to the second decimal.

\begin{tabular}{|c|c|c|c|c|}
\hline \multicolumn{5}{|c|}{$\begin{array}{c}\text { Comparison of the LPQRD and } \\
\text { Longman methods }\end{array}$} \\
\hline & $\begin{array}{c}\text { Frequency } \\
(\mathbf{H z})\end{array}$ & $\begin{array}{l}\text { Decay } \\
\text { time }(\mathrm{s})\end{array}$ & Amplitude & $\begin{array}{l}\text { Phase } \\
\text { (degrec) }\end{array}$ \\
\hline \multicolumn{4}{|l|}{ Theoretical } & 0.0 \\
\hline LPQRD & 1.10 & 1.00 & 2.00 & 0.0 \\
\hline \multirow[t]{2}{*}{$M=5$} & 1.01 & 2.47 & 0.85 & -16.4 \\
\hline & 1.10 & 1.08 & 2.19 & 5.8 \\
\hline \multirow[t]{2}{*}{$M=10$} & 1.00 & 1.95 & 1.04 & 0.7 \\
\hline & 1.10 & 1.00 & 1.96 & -0.4 \\
\hline \multirow[t]{2}{*}{$M=20$} & 1.00 & 1.95 & 1.06 & -0.5 \\
\hline & 1.10 & 1.00 & 1.94 & 0.2 \\
\hline \multicolumn{5}{|l|}{ Longman's } \\
\hline \multirow{2}{*}{$M=5$} & 1.05 & 1.24 & 2.78 & -0.3 \\
\hline & 1.33 & 0.46 & 0.28 & -4.1 \\
\hline \multirow[t]{2}{*}{$M=10$} & 1.01 & 1.62 & 1.47 & 4.0 \\
\hline & 1.12 & 0.93 & 1.55 & -3.5 \\
\hline \multirow[t]{2}{*}{$M=20$} & 1.01 & 2.21 & 0.95 & 6.1 \\
\hline & 1.10 & 1.07 & 2.09 & -13.5 \\
\hline
\end{tabular}

In this test we compared both methods with a $z$-transform expansion at infinity, as in the conventional Prony method. They all failed to resolve two components with $M=3$ (not listed in table), and the Longman method still failed with $M=5$.

As nicely illustrated by Yeramian and Claverie', in many cases better accuracy can be obtained if the Taylor expansion of the Laplace integral can be made at some points other than with a $z_{0}$ at infinity. Similarly, within the framework of the LP and $z$-transform ${ }^{3}$ theory, the matrix equation of the linear prediction can be slightly modified so that the LP coefficients are evaluated with $z$-transfrom in the neighbourhoods of the actual roots. The improvement of the resolving power and numerical accuracy in either their Laplace transform or our $z$-transform depends on how closely one can estimate the expansion centre or the actual roots. In the Padé method the Taylor series expansion of the Laplace integral can be made only at one particular point. Thus, clusters close to the expansion centre may be better resolved, but distant clusters may still remain unresolved. In contrast, the LP approach is preferable because the $z$-transform can be evaluated simultaneously around several clusters, resulting in a more global improvement in resolution. Finally, the Laplace integral transform is similar to $z$-transform, except that the former is in a continuous and the latter is in a discrete form. As most experimental data are recorded as discrete points, the $z$ transform seems to be more appropriate. Comparing the stability between the Longman method and the matrix decomposition method with a least-squares fit, the LP routine using QRD or SVD appears to be the method of choice.

\section{J. TANG}

J.R. NORRIS

Chemistry Division,

Argonne National Laboratory,

Argonne, Illinois 60439, USA

1. Yeramian, E. \& Claverie, P. Nature 326, 169-174 (1987)

2. Prony, R. J. Ecole Polytechnique 1, 24-76 (1795)

3. Tang, J. \& Norris, J.R. J. chem. Phys. 84, 5210-5211 (1986)

4. Tang, J., Lin, C.P., Bowman, M. K. \& Norris, J.R. J. magn Tang, J., Lin, C.P., Bowm

5. Barkhuijsen, H., De Beer, J., Bovee, W.M.M.J. \& Van Ormondt, D. J. magn. Reson. 61, 465-481 (1985)

6. Longman, I.M. Int. J. Computer Math. B3, 53-64 (1971).

\section{How fecundity balances mortality in birds}

SIR-Birth rates must balance death rates if natural populations are to persist. Saether demonstrated that two components of fecundity, clutch size and age at maturity, help compensate for differences in mortality among European bird species. But fecundity also depends on a third variable, the number of clutches per year, for which data are available ${ }^{2}$ on Saether's 107 species. Following Saether, we performed analyses at the family level ( $n$ $=34$ ). Number of clutches per year decreases with increased survival rate $(r=$ $-0.634, P<0.001$, and thus also compensates for mortality. Annual fecundity, the product of clutch size and number of clutches, is even more closely correlated with survival $(r=-0.824, P<0.001)$.

Saether' also considered the relationship of clutch size and age at maturity to each other and to survival after the effects of body size had been removed by partial correlation analysis. He found that families with larger clutches also have earlier ages at maturity when body weight is controlled for. We find that number of clutches per year is not significantly correlated with clutch size $(r=0.143)$ but is negatively correlated with age of maturity ( $r=$ $-0.478, P<0.01)$ and with survival $(r=$ $-0.374, P<0.05$ ) when body weight is partialled out. But clutch size is not significantly correlated with body weight across the sample of European birds ( $r=$ $-0.284)$, whereas both number of clutches per year and age at first breeding are significantly correlated with body weight ( $r=$ $-0.658,0.614$, both $P<0.001$ ).

Similar patterns of covariation among the fecundity factors and body weight occur in a much larger sample of 3,142 species from 149 families ${ }^{2}$. We agree with Saether that the cause of such differences may be evolutionary or a direct result of density-dependent processes.

Peter M. Bennett

Institute of Zoology,

Zoological Society of London,

London NWI 4RY, UK

Paul H. Harvey

University of Oxford, Oxford OX1 3PS, UK

1. Saether, B.-E. Nature 331, 616-617 (1988).

2. Bennett, P. M. thesis, Univ. Sussex (1986).
Department of Zoology, 